\title{
Bubble plumes and the Coanda effect
}

\author{
Atila P. Silva Freire ${ }^{\text {a,* }}$, Davi D’E. Miranda ${ }^{\text {b }}$, Leonardo M.S. Luz ${ }^{\text {, }}$, \\ Guilherme F.M. França ${ }^{b}$ \\ ${ }^{a}$ Mechanical Engineering Program (PEM/COPPE/UFRJ), Federal University of Rio de Janeiro, C.P. 68503, \\ 21945-970 Rio de Janeiro, Brazil \\ ${ }^{\mathrm{b}}$ Department of Mechanical Engineering (DEM/EE/UFRJ), C.P. 68529, 21945-970 Rio de Janeiro, Brazil
}

Received 7 May 2001; received in revised form 27 March 2002

\begin{abstract}
This paper describes the mean gas fraction distribution in the two-phase flow of a gas-liquid bubble plume set to develop adjacent either to a wall or to another bubble plume. When this happens, the plume exhibits a type of Coanda effect, bending either towards the wall or the other plume. The local mean gas fraction measurements are carried out using the electro-resistivity probe technique in an air-water system. The deflection angle of the plumes is shown to present a dependence on the modified Weber and Froude numbers of the bubbles. A Gaussian distribution for the mean gas fraction profile, observed to exist for axisymmetric single plumes, is shown not to occur in flow geometries where the Coanda effect is allowed to set in. The transition zone between the downstream flow where two Gaussian plumes are observed and the far upstream flow where the plumes are seen to merge onto a single plume is characterized. Photographs of the flow are shown to illustrate the phenomenon. Results are present for two- and three-dimensional mean gas fraction distributions. In addition, a simple theory based on integral methods is advanced for the prediction of the plume deflection angle; the theory considers a variable entrainment co-efficient.
\end{abstract}

(C) 2002 Elsevier Science Ltd. All rights reserved.

Keywords: Bubble plume; Coanda effect; Entrainment

\section{Introduction}

Buoyant plumes in multi-phase flows are a common occurrence in the chemical and metallurgical industries. Indeed, bubble plumes provide an effective and relatively simple way of

\footnotetext{
"Corresponding author.

E-mail address: atila@serv.com.ufrj.br (A.P.S. Freire).
} 
achieving large degrees of agitation in the flow of fluids which are otherwise hostile to intrusive elements.

For example, in the steel making industry, gas stirred melt ladles are common place. The upward movement of an inert gas into a bath provokes a large agitation in the molten metal, resulting in the chemical and thermal homogenisation of the mixture, as well as in an acceleration of the absorption of harmful non-metallic inclusions into an overlaying slag.

The liquid metal homogenisation can indistinctly be performed by a single gas plume, by a combination of geometrically arranged plumes or by a curtain of bubbles. The degree of agitation - and consequently the flow properties - provided by rising bubbles of an inert gas in the liquid metal medium must hence be determined so as to achieve minimum mixing times and maximun recoveries of alloy additions at optimum flow rates.

The flow properties will, of course, depend on the geometry of the problem and, in particular, on the specific manner in which gas is injected in the considered vessel. For most studies in literature, just a single source of gas is considered, which is taken to be axisymmetric. This is, in fact, a mandatory procedure when an integral approach to the problem is used and the entrainment hypothesis considered. For differential approaches, this need not be the case; turbulence models with a reasonable degree of generality should be capable of accounting for the break in symmetry so that, in theory, one or more plumes could be considered in the analysis.

The purpose of this paper is to investigate the behaviour of bubble plumes that develop near to a wall or near to another bubble plume. Under this condition the plume is seen to develop not axisymmetrically, but deflected sideways. The emphasis here is on understanding how the proximity of the wall, or of another plume, deflects the plume, altering its mean properties. As a bonus, the paper develops a simple integral theory to describe the phenomenon. The theory is an extension of the theory of Ditmars and Cederwall (1974), making use, for the sake of simplicity, of Gaussian profiles and of Boussinesq assumption.

Here, a comment seems in order. The flow geometries to be studied should exhibit the same type of physical effects as those that appear in the case of thermal plumes. The effect of an adjacent wall on the inclination of a plume is about the same as the effect of another plume of equal strength and twice as far away (Pera and Gebhart, 1975). Thus, through the artifice of studying the plume development close to a wall, one may expect to understand the problem of multiple plumes which develop close enough to each other to have their original axisymmetry broken. The present work makes a detailed comparison between plumes that develop near to a wall and plumes that develop close to another plume, to assess if the trends observed for thermal plumes are repeated for bubble plumes. In particular, the statement related to the equivalent effects of an adjacent wall and an adjacent plume will be carefully investigated.

To measure the main characteristics of the plumes, an electro-resistivity probe system was used. This system was chosen for being simple while still being capable of conveying much useful information on the phenomenon; it will be discussed in detail in an appropriate section. Here, it suffices to say that by an analysis of the experimental data through the mean gas fraction profiles, the deflection of the plume has been evaluated as a function of several parameters of interest including the gas flow rate and the distance between the point sources.

The single bubble plume flow has been extensively studied both theoretically (Ditmars and Cederwall, 1974; Milgram, 1983; Brevik and Killie, 1996) and experimentally (Milgram, 1983; Castillejos and Brimacombe, 1987; Barbosa and Bradbury, 1996) by many authors in the past 
three decades. The studies cover a large range of conditions but fail to propose a single model capable of dealing with all possible variations in flow conditions. For the integral approaches, the flow has been divided into three distinct regions where some dominant effects prevail (Milgram, 1983). The buoyancy-dominated region is normally referred to as the "zone of established flow". This will be the region of main interest here.

For flow geometries where two or more plumes are present, the number of works is considerably smaller. A relatively complete work on multiple plume systems is the work of Joo and Guthrie (1992). In this work, the twin bubble plume arrangement flow has been studied both from an experimental and a numerical point of view. Using a tracer technique to quantify the total mixing time - the time interval that $95 \%$ of the tracer needs to be mixed in the flow-several plume arrangements were tested. The flow pattern for these arrangements were then numerically reproduced with the help of a $\kappa-\epsilon$ turbulence model. In all numerical calculations, the plumes presented a clear upright position, irrespective of their proximity to the wall. Thus, no sign of the Coanda effect was observed in the numerical computations. Some pictures of the phenomenon, taken by the same author, were however very clear in exhibiting the plume's deflection. The mathematical modelling of the flow was, therefore, in clear disagreement with the experimental evidence. In this simulation, the action of the bubbles was limited only to the buoyancy term, resulting in a very simple model for the gas phase that could not predict the interaction between the plumes.

As far as the interaction between adjacent bubble plumes is concerned, no analysis (experimental or theoretical) has been identified by the present authors in literature. The interaction between laminar thermal plumes has been studied using a Mach-Zehnder interferometer (Pera and Gebhart, 1975). For this case, a simple model was developed in order to take into account the deflection angle of the centre lines of the plumes. Pera and Gebhart observed that plane plumes manifest stronger centre line inclinations than axisymmetric plumes.

\section{The Coanda effect}

The Coanda effect is the tendency of a fluid, either gaseous or liquid, to cling to a vertical surface that is near to an orifice from which the fluid emerges (Reba, 1966).

An easily verified experimental fact is that when two point sources of buoyancy (or of momentum) are placed side by side, the two resulting turbulent plumes (or jets) tend to bent towards each other. In this case, the bending of the plumes is not motivated by a pressure difference, as observed when the resulting plumes are laminar (Pera and Gebhart, 1975). When the flow is turbulent, the plumes bend due to a restriction in the entrainment of external fluid by the mean flow. Particularly in the axisymmetric geometry, the role played by pressure difference is negligible when compared with that of turbulent entrainment. Despite the difference in the nature of the forces that govern each phenomenon, some authors still consider both (pressure difference and entrainment restraint) as examples of the Coanda effect.

In the present work, the authors suggests that the bending of a turbulent round bubble plume is caused by a momentum flux unbalance related to the entrainment restraint due to the presence of an adjacent wall or of another plume. The analysis in the subsequent sections of this paper will be entirely based on this premise. 


\section{Experiments}

\subsection{The experimental technique}

Electro-resistivity sensors built from small needles were simultaneously developed by Neal and Bankoff (1963) and Nassos (1963). In these studies, almost all efforts were dedicated to the development of the experimental technique rather than to the investigation on the nature of some particular type of flow. Neal and Bankoff used a nitrogen-mercury system, whereas Nassos carried out measurements in an air-water system. Chesters et al. (1980) were the first to employ the resistivity technique in gas-liquid non-confined flows with a certain degree of success. The authors used electro-resistivity sensors together with laser-Doppler anemometry to describe the characteristics of the liquid and the gas phases in a bubble plume. Tacke et al. (1985), studying gas stirred steel making processes, used the electro-resistivity sensor technique to make some measurements of the gas-phase properties in air-water, helium-water and nitrogen-mercury systems. Castillejos and Brimacombe (1987), also aiming at the application of the bubble plume phenomenon in the steel making industry, developed a comprehensive instrumentation based on the resistivity technique to investigate the problem. Teyssedou et al. (1988) presented a new AC probe system, together with an analysis of the effect of the geometry of the sensor tip and of other parameters on the performance of the system. More recently, Kocamustafaogullari and Wang (1991), Leung et al. (1992) and Liu and Bankoff (1993) used resistivity sensors to determine local time-averaged mean gas fraction, interfacial area concentration, bubble rise velocity and bubble pierced length in internal bubbly flows.

The working principle of the experimental technique is based on the difference between the electrical conductivity (resistivity) of the phases. Since the electrical conductivity of water is much higher than that of air, it is assumed, for practical purposes, that only the continuous phase (liquid) is capable of conducting electrical current. Accordingly to Herringe and Davis (1974), resistivity sensors are the most suitable technique for measurements in two-phase mixtures where the continuous phase is conductive. The main adversity of the technique is the existence of an instream sensor, which affects the structure of the flow.

In a double channel system (whether AC or DC supply), the difference in electrical resistivity between the phases can be sensed by the electrodes in the two-phase flow so that parameters like the local time-averaged mean gas fraction, the rise velocity and the pierced length of bubbles can be obtained through an analysis of the output signal.

\subsection{The experimental apparatus}

The measuring system used in this work has been fully described in Barbosa and Bradbury (1996) and Barbosa (1997); for any detailed information on the system the reader is, therefore, referred to those works. Next we will just briefly comment on the features of the probes.

Co-axial probes were chosen to be used here. In fact, due to its symmetric geometry, the coaxial probe interference on the flow is recognized as being weaker than that of a parallel probe of nearly equal dimensions. The measuring probes were constructed with the following features: (i) $0.2 \mathrm{~mm}$ diameter stainless steel internal wire (upstream electrode); (ii) $0.4 \mathrm{~mm}$ OD $0.2 \mathrm{~mm}$ ID hypodermic 
tubing (downstream electrode); (iii) length of electrodes free of insulation equal to $0.1 \mathrm{~mm}$; and (iv) distance between electrodes equal to $1.5 \pm 0.15 \mathrm{~mm}$.

The experimental apparatus is shown in Fig. 1; it comprises a water tank, an air injection system, a two-dimensional traversing mechanism and a data acquisition and analysis system. The glass water tank had dimensions $1 \times 1 \times 1 \mathrm{~m}$ and was filled with a $3 \mathrm{~g} / \mathrm{l}$ sodium chloride solution (brine). The air injection system was composed of a mass flow meter and two injection nozzles with a single $3.2 \mathrm{~mm}$ diameter hole. The data acquisition and analysis system consisted of a microcomputer with an interface data acquisition board, an oscilloscope, a signal conditioner module and the electro-resistivity probes.

The probe was placed perpendicularly to the tank bottom. The water depth was kept constant and equal to $0.85 \mathrm{~m}$. Table 1 summarises the experimental conditions examined.

The non-dimensional groups are defined accordingly to

$$
F_{\mathrm{r}}=\frac{q^{2}}{g s^{5}}, \quad W_{\mathrm{e}}=\frac{\Delta \rho q^{2}}{\sigma s^{3}},
$$

where $g$ is the acceleration of gravity, $q$ is the gas flow rate issuing from each source, $\sigma$ is the surface tension and $\Delta \rho$ is the density difference between the phases. The quantities above are, respectively, the modified Froude, $F_{\mathrm{r}}$, and Weber, $W_{\mathrm{e}}$, numbers based on the halved distance between the sources, $s$, or the distance from the wall.

The mean gas fraction at a point in the flow is a time-averaged property given by

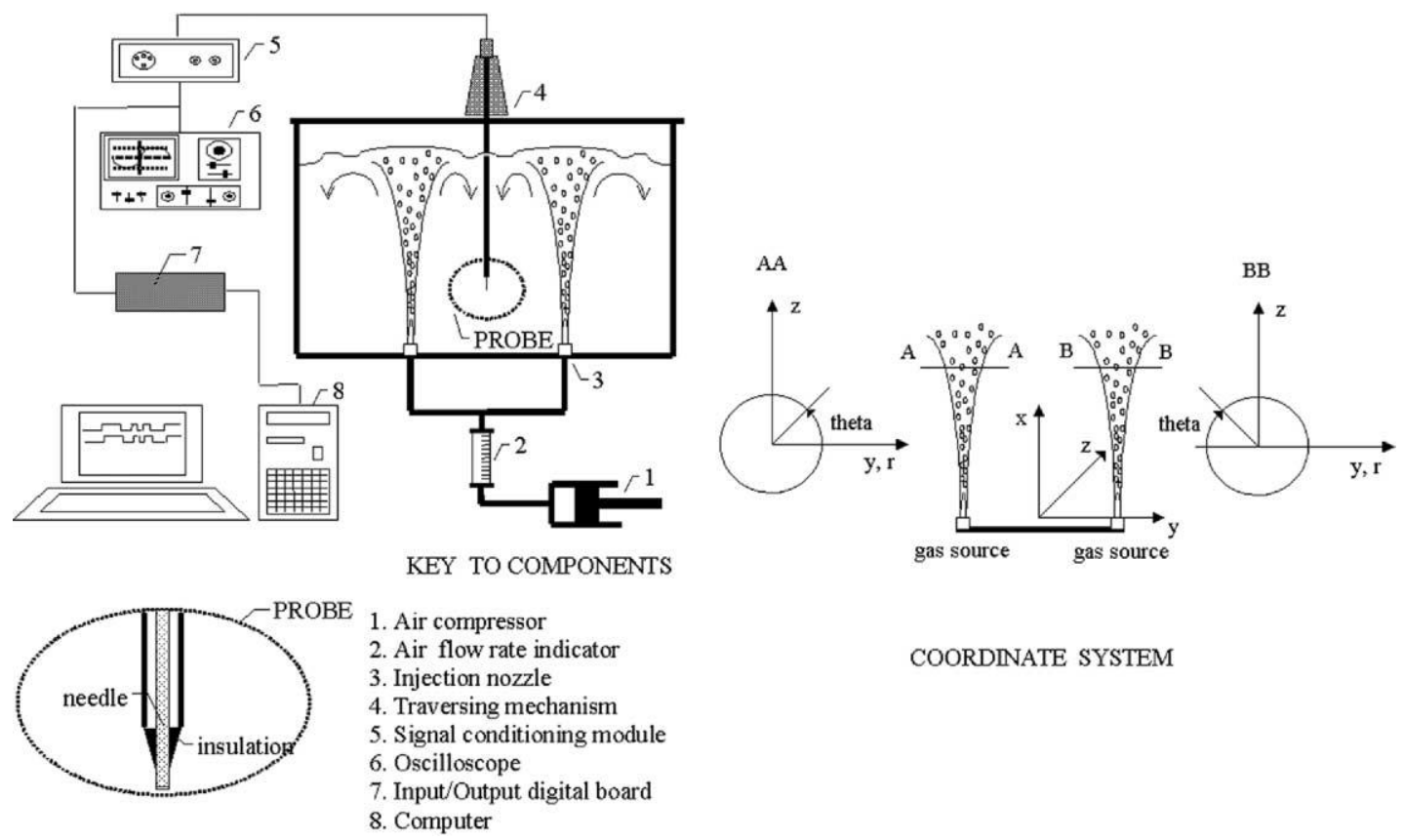

Fig. 1. Experimental set-up and co-ordinate system. 
Table 1

Experimental test conditions

\begin{tabular}{lllll}
\hline Case & $s(\mathrm{~cm})$ & $q(1 / \mathrm{min})$ & $F_{\mathrm{r}}$ & $W_{\mathrm{e}}$ \\
\hline Plume near a wall & & & & \\
$\mathrm{a}$ & 2 & 1.2 & $1.13 \mathrm{e}-3$ & 1.1594 \\
$\mathrm{~b}$ & 3.15 & 2 & $3.65 \mathrm{e}-3$ & 0.8877 \\
$\mathrm{c}$ & 3.15 & 3 & $8.22 \mathrm{e}-3$ & 1.9972 \\
$\mathrm{~d}$ & 6.1 & 2 & $1.34 \mathrm{e}-4$ & 0.1222 \\
$\mathrm{e}$ & 6.1 & 3 & $3.02 \mathrm{e}-4$ & 0.2750 \\
$\mathrm{f}$ & 9 & 1.2 & $6.91 \mathrm{e}-6$ & 0.0137 \\
$\mathrm{~g}$ & 9 & 3.5 & $5.87 \mathrm{e}-5$ & 0.1166 \\
$\mathrm{~h}$ & 13 & 1.2 & $1.10 \mathrm{e}-6$ & 0.0045 \\
Two plumes & & & & \\
$\mathrm{i}$ & 3.25 & 1.2 & $1.12 \mathrm{e}-3$ & 0.2910 \\
$\mathrm{j}$ & 3.25 & 2.4 & $4.50 \mathrm{e}-3$ & 1.1638 \\
$\mathrm{k}$ & 6.4 & 1.2 & $3.80 \mathrm{e}-5$ & 0.0381 \\
$\mathrm{l}$ & 6.4 & 2.4 & $1.52 \mathrm{e}-4$ & 0.1524 \\
$\mathrm{~m}$ & 9.05 & 1.2 & $6.72 \mathrm{e}-6$ & 0.0135 \\
$\mathrm{n}$ & 9.05 & 2.4 & $2.69 \mathrm{e}-5$ & 0.0539 \\
\hline
\end{tabular}

$s$ : halved distance between the gas sources; $q$ : gas flow rate. $F_{\mathrm{r}}$ and $W_{\mathrm{e}}$ denote respectively the modified Froude and Weber numbers; they are defined by Eq. (1).

$$
f(r, x)=\frac{1}{T} \int_{0}^{T} I(r, x, t) \mathrm{d} t
$$

where $T$ is the total sampling time, $I$ is the digital output signal from the conditioning module and $r$ and $x$ are the co-ordinates shown in Fig. 1. The output signal, $I$, consists of a series of pulses which correspond to the transit of bubbles through the probe. Further details concerning the output signal, $I$, are available in Barbosa and Bradbury (1996) and Barbosa (1997).

The data were acquired at a sampling rate of $\approx 2.5 \mathrm{kHz}$ and about 50 sampling blocks of 10,000 readings (Barbosa and Bradbury, 1996; Barbosa, 1997) were shown to be sufficient to describe the flow at each measured point. In fact, in Barbosa (1997), the shape, size and velocity of the rising bubbles was detailed studied; also, the influence of the injection geometry on plume development and the existence of any lateral wandering motion of the plume were investigated.

The probe calibration was carried out in a vertical pipe with $4 \mathrm{~cm}$ diameter and $100 \mathrm{~cm}$ length. After the pipe had been filled with water taken from the tank and the probe had been placed in position, a large bubble was carefully introduced at its bottom through a syringe. As the bubble raised and hit the probe, the recordings from a Panasonic video camera were digitalized and analysed. The pictures which were taken at a frequency of 60 frames/s were then compared with the signal of the conditioning module. The resistance of the flow in the conditioning module was then set so as to furnish the same response in both systems.

An uncertainty analysis of the data was performed according to the procedure described in Kline (1985). Typically the uncertainty associated with the mean gas fraction measurements was: $f=0.00035$ precision, 0 bias $(P=0.95)$. 


\section{Results}

\subsection{Two-dimensional data}

For plumes that develop near to a wall or near to another plume, the general flow pattern for both axisymmetric plumes and bent plumes is shown in Figs. 2 and 3 respectively. In the pictures, the bending of the plumes is clearly illustrated; in fact, and within the experimental conditions of the present work, the plumes are observed to assume a very steady flow pattern, confirming what had been observed by Barbosa and Bradbury (1996) and Barbosa (1997) for an axysimmetric plume. The photographs shown at Figs. 2 and 3 were taken with long exposition times so that they illustrate the mean position of the plumes.

Results for one plume near a wall are presented in Figs. 4-6.

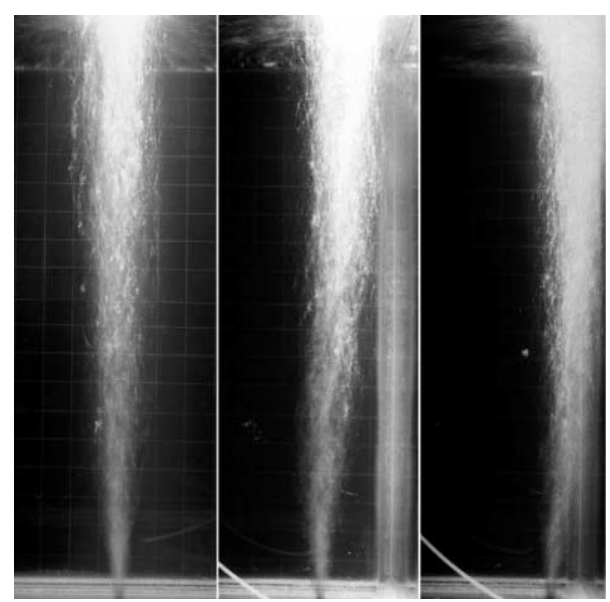

Fig. 2. General flow pattern for one plume close to a wall.

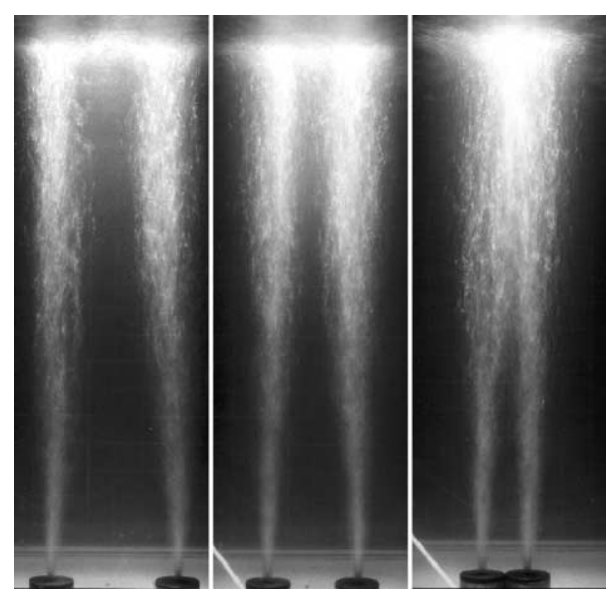

Fig. 3. General flow pattern for two plumes. 


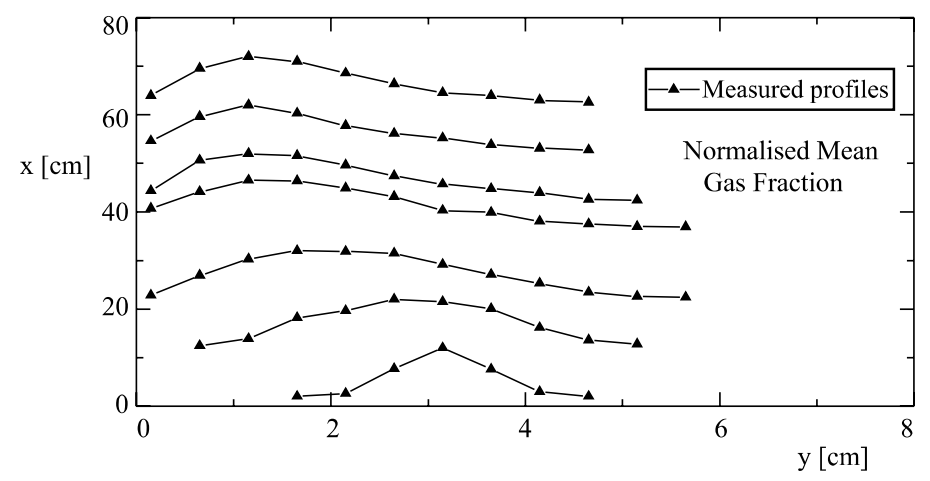

Fig. 4. Local mean gas fraction profiles for several $x$-stations, $s=3.15 \mathrm{~cm}, q=21 / \mathrm{min}$. The origin $y=0$ is the wall location. The mean gas fraction is normalized against the centerline value.

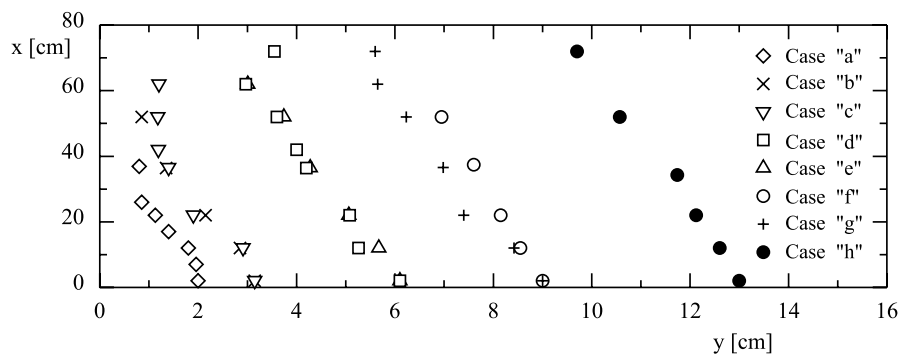

Fig. 5. Deflection of the plume for several gas flow rates and distances from the wall. The origin $y=0$ is the wall location.
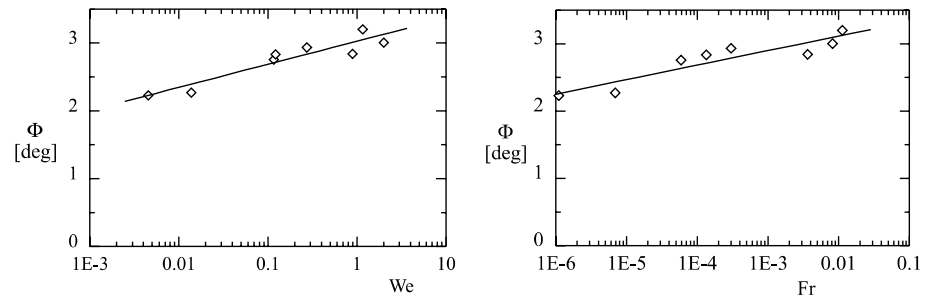

Fig. 6. Deflection angle versus Weber and Froude numbers.

Fig. 4 shows local mean gas fraction profiles for several $x$-stations. The first two profiles near to the gas source have a Gaussian form, much in the same way as for axisymmetric plumes. The points of maximum mean gas fraction can easily be identified in the figure. They will be taken as our reference for the evaluation of the position of the centre line of the plume. From previous studies, we know these points to correspond to the points where the liquid-phase velocity is maximum as well.

The deflection of the plume for several gas flow rates and distances from the wall are shown in Fig. 5 in dimensional coordinates. This figure shows that most of the trajectories can reasonably 
be approximated by a straight line. For the plumes whose distance from the wall is large this trend is particularly well defined. We may thus construct graphs for the variation of the deflection angle defined by these straight lines as a function of the Froude and the Weber numbers. These graphs are shown in Fig. 6; they show that $\phi$ increases logarithmically and monotonically with both $F_{\mathrm{r}}$ and $W_{\mathrm{e}}$ according to the two displayed curves.

Results for the double plume arrangement are presented in Figs. 7-9.

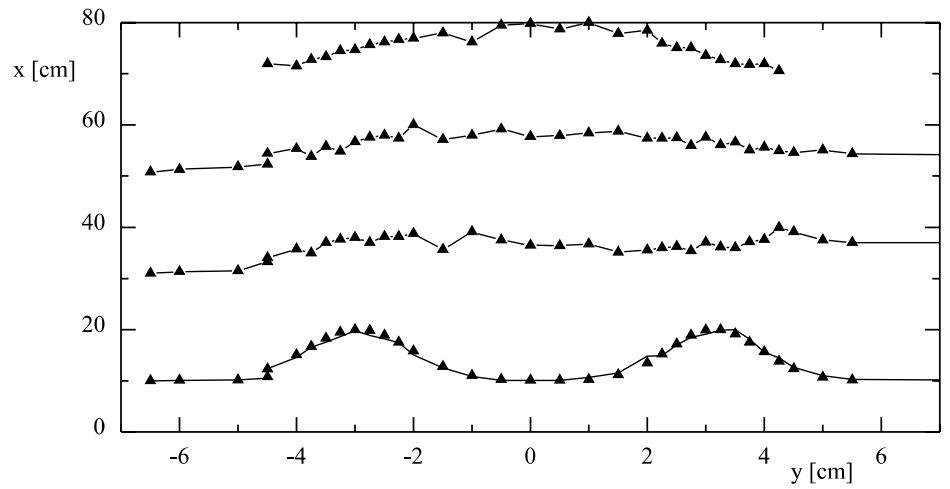

Fig. 7. Local mean gas fraction profiles for several $x$-stations, $s=3.15 \mathrm{~cm}, q=21 / \mathrm{min}$. The origin $y=0$ is the midpoint between the gas sources. The mean gas fraction is normalized against the centerline value.

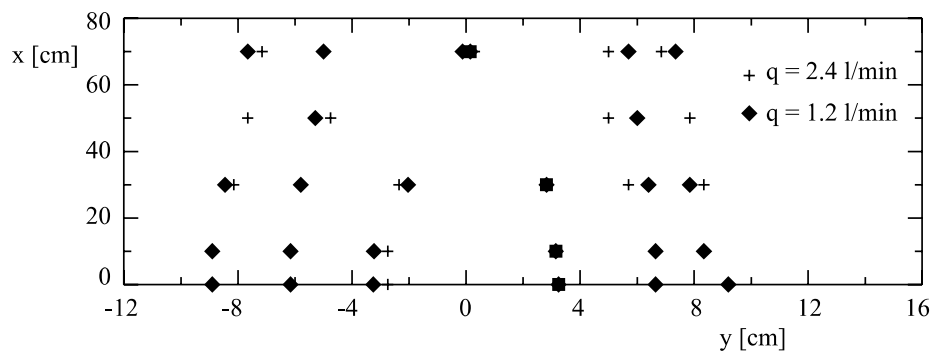

Fig. 8. Deflection of the plume for several gas flow rates and distances from the wall. The origin $y=0$ is the midpoint between the gas sources.
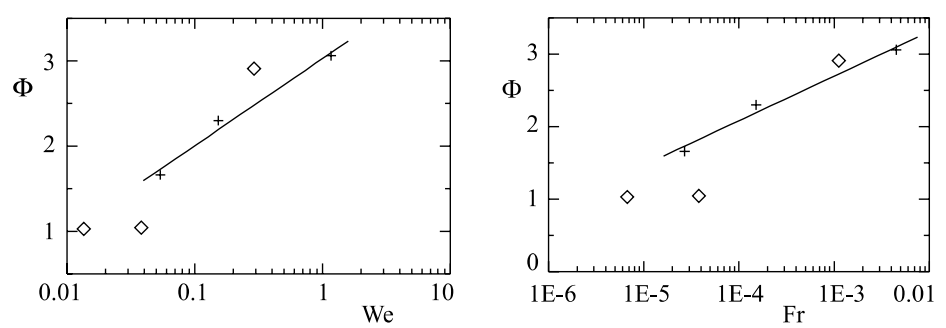

Fig. 9. Deflection angle versus Weber and Froude numbers. 
The shape of the deflected plume is shown in Fig. 3. Note that the plume deflection is easily observed even through a casual observation of the photographs. The mean gas fraction profiles at different $x$-stations are shown in Fig. 7. The profile corresponding to the lowest station presents well defined Gaussian curves showing that the plume interaction cannot still be felt at this height. All subsequent profiles, however, present a noticeable deformation due the proximity of the other plume. The points of maximum mean gas fraction are seen to migrate to the centre of the geometrical arrangement, giving origin well downstream to a single plume with a well defined Gaussian shape. The points of maximum mean gas fraction correspond as well to the points of maximum velocity, being, therefore, used to define the centre of the plumes.

The deflection angle of the plumes is shown next in Fig. 8 for two different gas injection rates and three different separation distances between the nozzles. Similarly to the results shown in Fig. 5, the centreline trajectories of the plume define nearly straight lines. The same deflection angles are shown in Fig. 9 against the Froude and Weber numbers; this figure show that the deflection angle increases logarithmically with $F_{\mathrm{r}}$ and $W_{\mathrm{e}}$.

\subsection{Three-dimensional data}

In this section we will present some three-dimensional mean gas fraction profile data for one selected condition, case $\mathrm{i}$.

All three-dimensional data were obtained with the electro-resistivity sensor technique described before. The probe, however, had five independent wire tips that could be used to yield simultaneous readings. These wires had the form of a spear with five points. The plane defined by the wires was then traversed perpendicularly to the plane defined by the centerline of the plumes from one end to the other. The tips of the spear were constructed $20 \mathrm{~mm}$ distant from each other and were all independently calibrated.

Before considering the three-dimensional structure of the plumes, let us show measurements of mean gas fraction profiles taken in the plane defined by the centerline of the plumes. Figs. 10-13 show the results for stations $x=200,270,350$ and $420 \mathrm{~mm}$. These figures had to be include here because of the low resolution of Fig. 7 .

The mean gas fraction contour lines are shown in Figs. 14-17.

\section{Theory}

Mean-flow theories for bubble plumes are integral theories for which the forms of the radial distributions of the velocity and of the density deficiency between the plume and the surrounding fluid are considered to be known in advance. In fact, since the pioneering work of Kobus (1968) to the more recent theories, very little in terms of the formulation has changed. The recent theories have incorporated many novelties, but the basic formulation of the problem remains the same; the governing equations are constructed from the conditions of conservation of gas, conservation of liquid, and the change of momentum flux with buoyancy.

In the present work, the theory of Ditmars and Cederwall (1974) will be used as the basis of our developments. By allowing the entrainment coefficient to vary along the plume periphery, the 


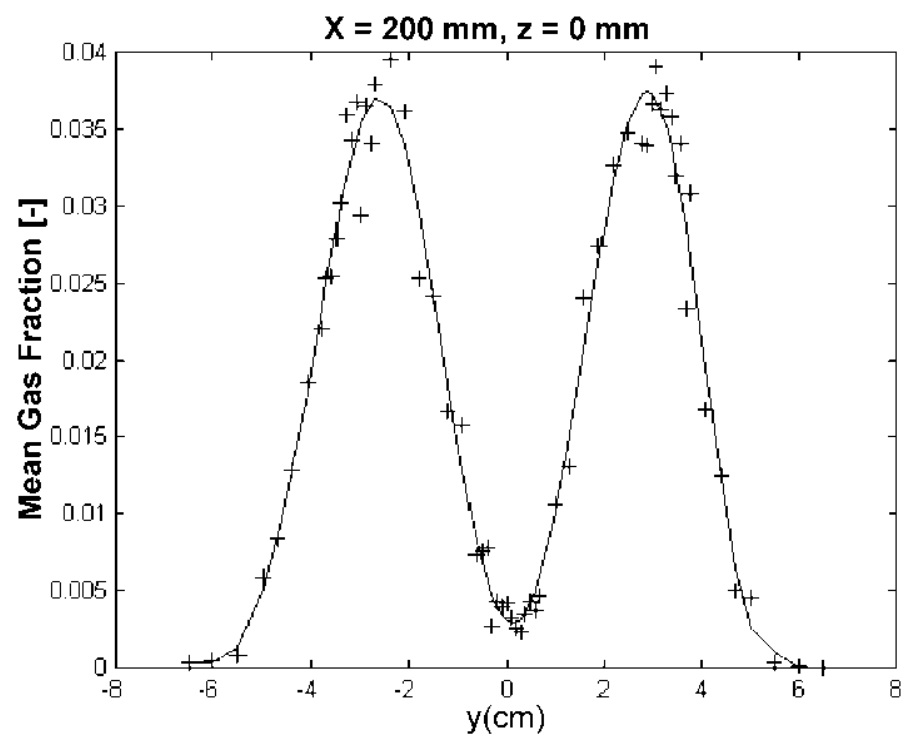

Fig. 10. Mean gas fraction profiles: $x=200 \mathrm{~mm}, z=0 \mathrm{~mm}$.

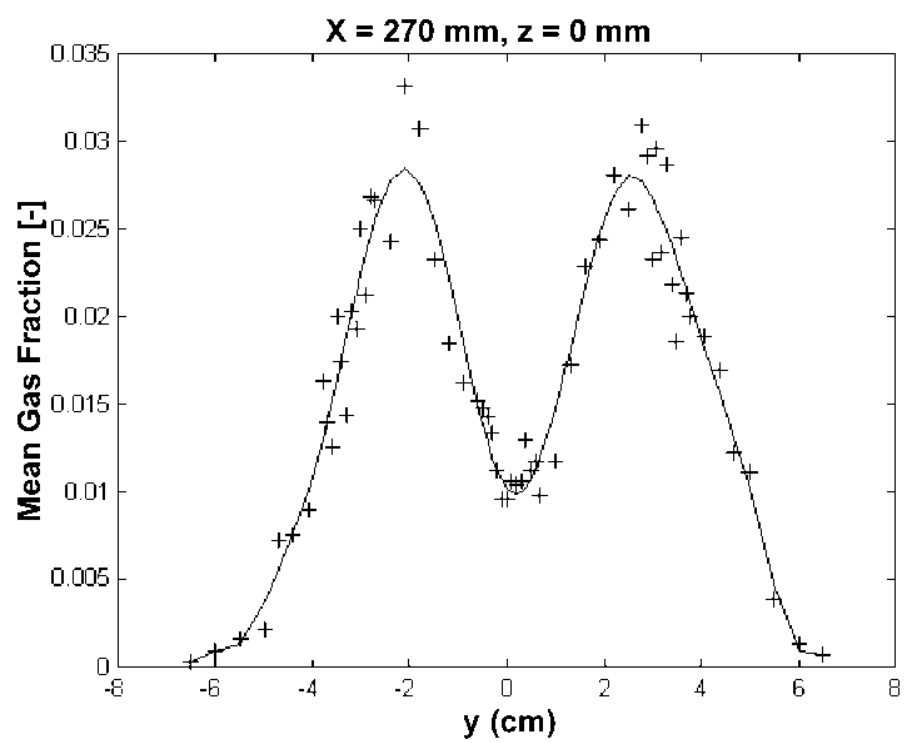

Fig. 11. Mean gas fraction profiles: $x=270 \mathrm{~mm}, z=0 \mathrm{~mm}$.

effect of deflection provoked by an adjacent twin plume can be conveniently modelled. This is a very simple premise, which yields very good results.

Since the basic details of the theory can be found in Ditmars and Cederwall, only a brief mention of them will be made here. A limitation of Ditmars and Cederwall's theory is its inability no capture any unsteadiness occurring in the flow. Thus, the plumes are considered stationary and free of lateral oscillations. 


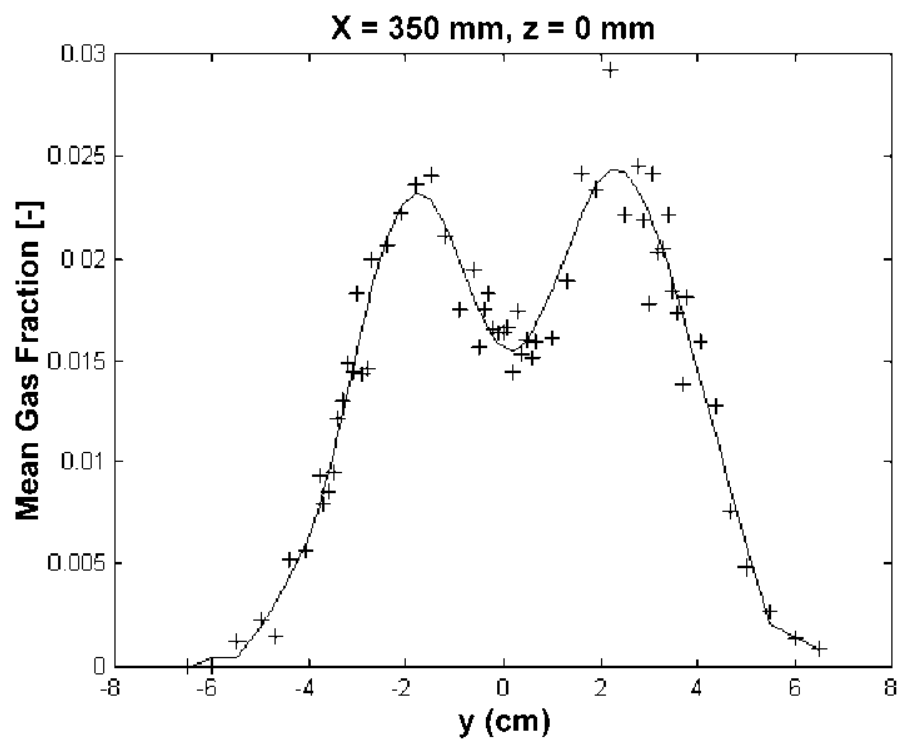

Fig. 12. Mean gas fraction profiles: $x=350 \mathrm{~mm}, z=0 \mathrm{~mm}$.

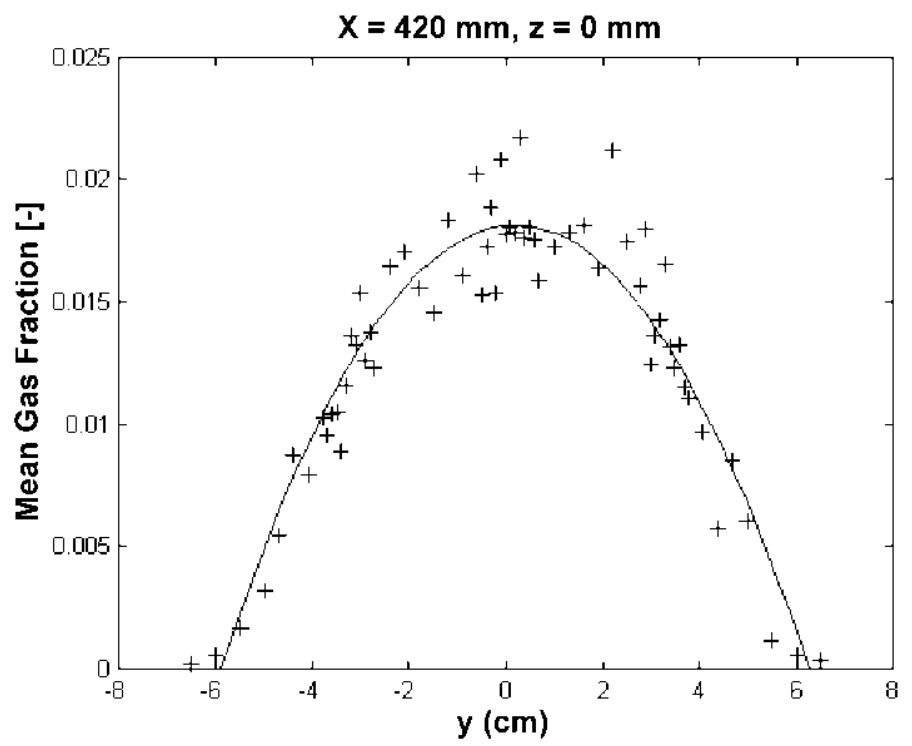

Fig. 13. Mean gas fraction profiles: $x=420 \mathrm{~mm}, z=0 \mathrm{~mm}$.

The velocity and mean density defect are given by

$$
\begin{aligned}
& u(r, x)=u_{\mathrm{m}}(x) \mathrm{e}^{-r^{2} / b^{2}}, \\
& \rho_{\mathrm{a}}-\rho_{\mathrm{m}}(r, x)=\Delta \rho_{\mathrm{m}}(x) \mathrm{e}^{-r^{2} /(\lambda b)^{2}},
\end{aligned}
$$




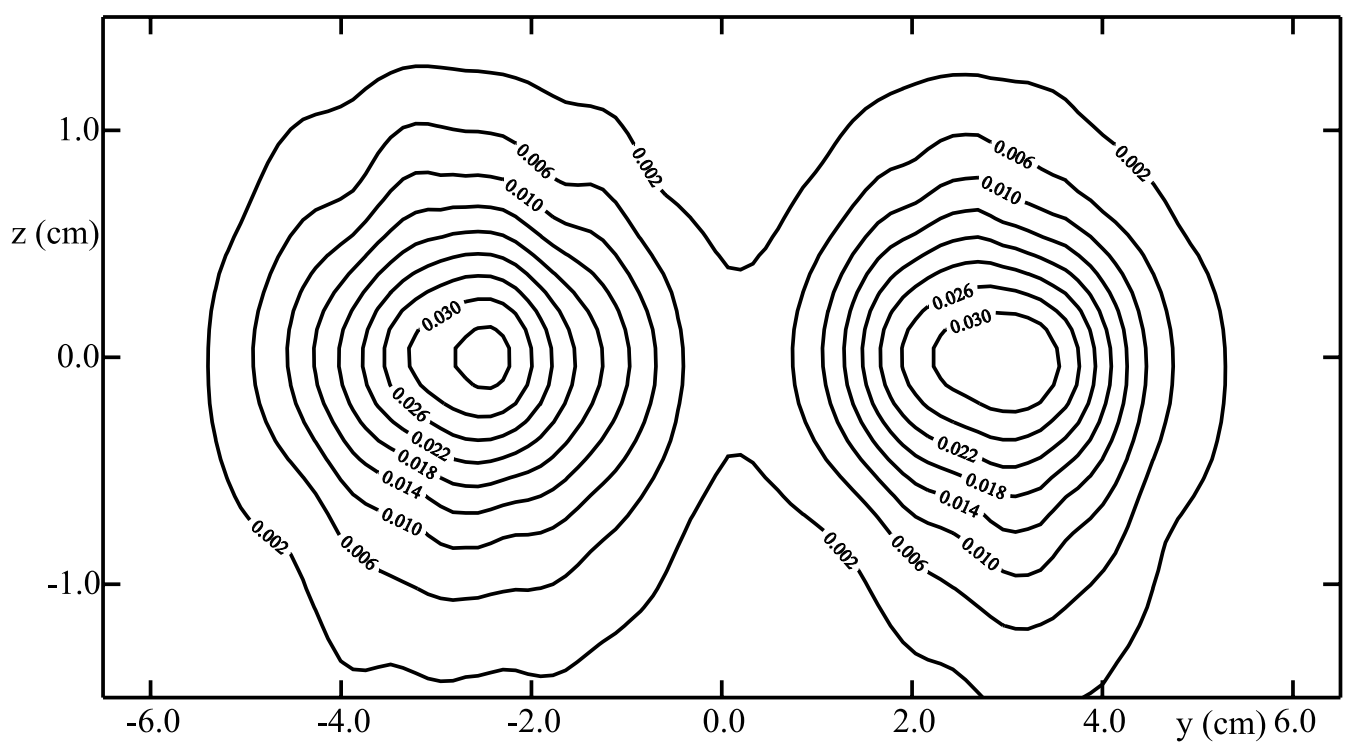

Fig. 14. Mean gas fraction contour lines: $x=200 \mathrm{~mm}$.

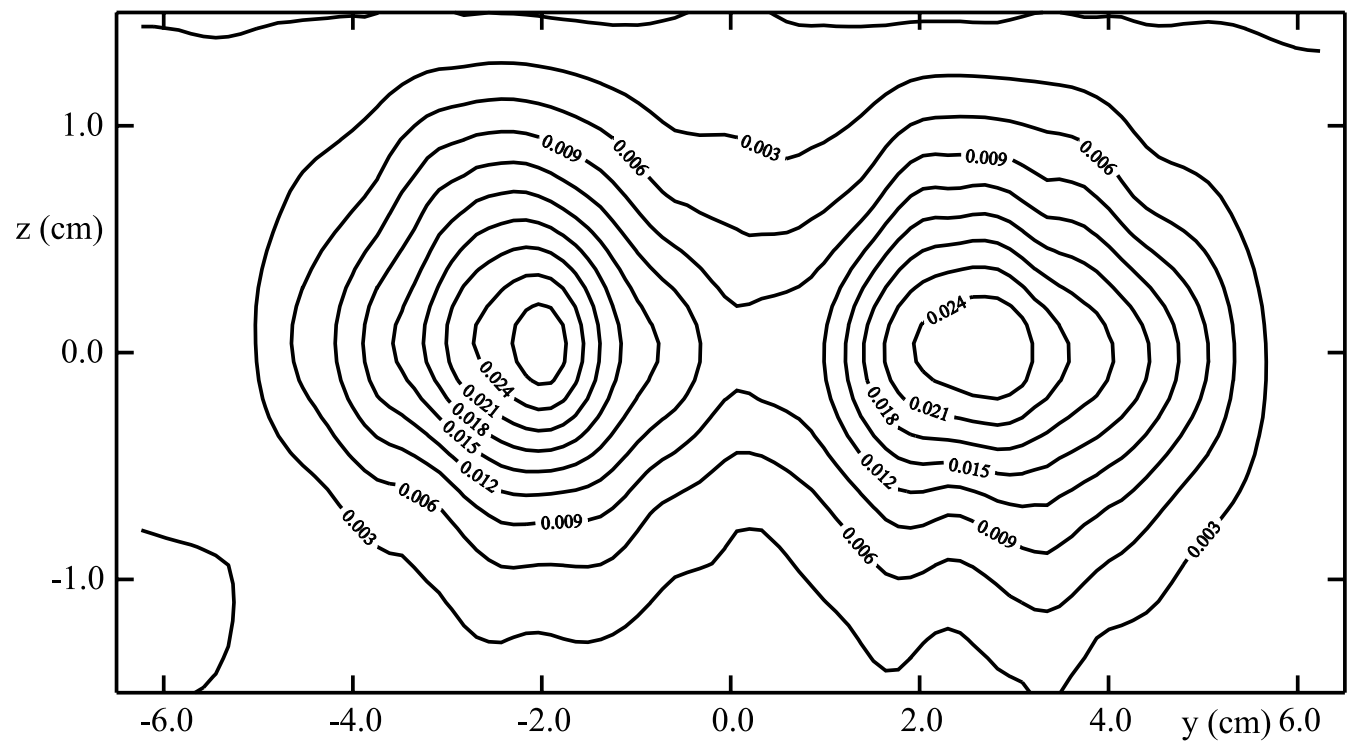

Fig. 15. Mean gas fraction contour lines: $x=270 \mathrm{~mm}$.

where $u_{\mathrm{m}}(x)$ is the centerline velocity, $b(x)$ is the characteristic lateral dimension of the plume related to the standard deviation of the lateral velocity distribution by $\sigma=b / \sqrt{2}, \rho_{\mathrm{m}}(x)$ is the local density of the air-water mixture, $\rho_{\mathrm{a}}$ is the density of water, $\Delta \rho_{\mathrm{m}}(x)$ is the centerline density difference between ambient water and air-water mixture within the plume at a particular level and $\lambda$ is the lateral spread of density deficiency to momentum. 


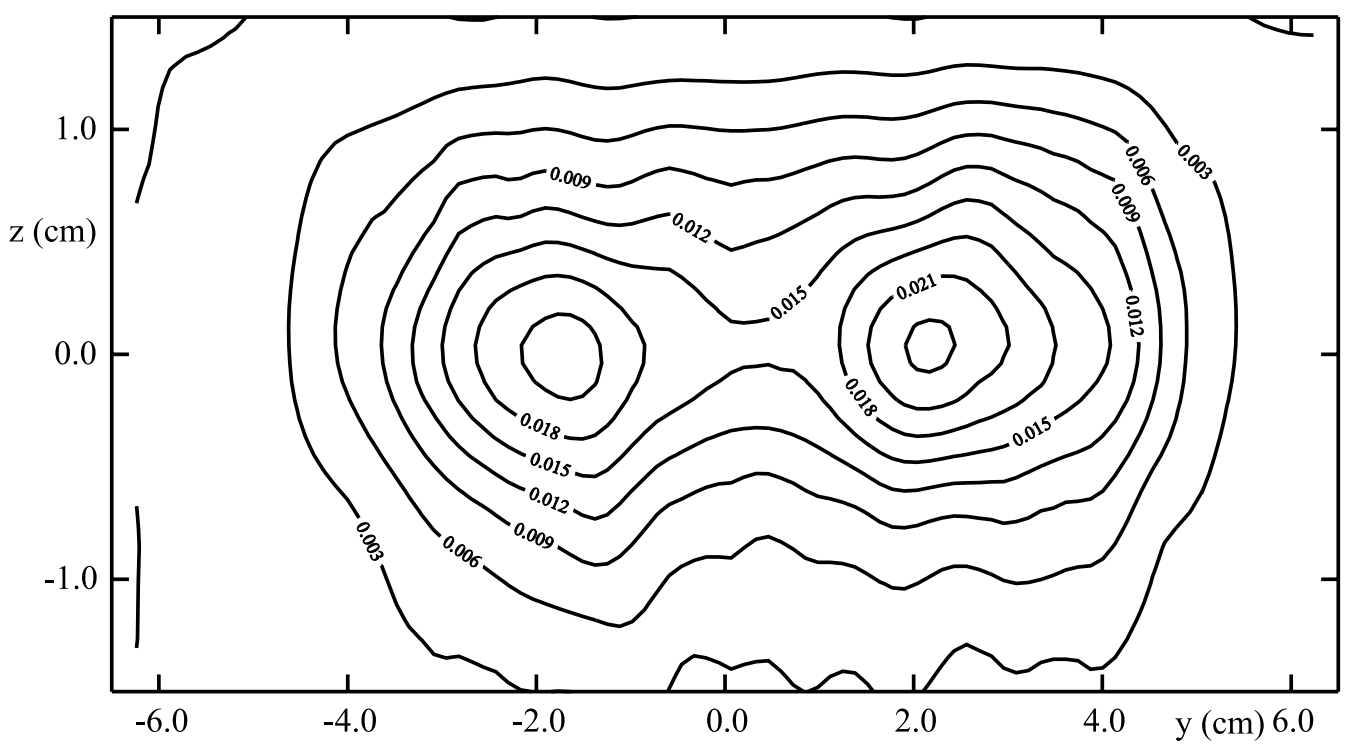

Fig. 16. Mean gas fraction contour lines: $x=350 \mathrm{~mm}$.

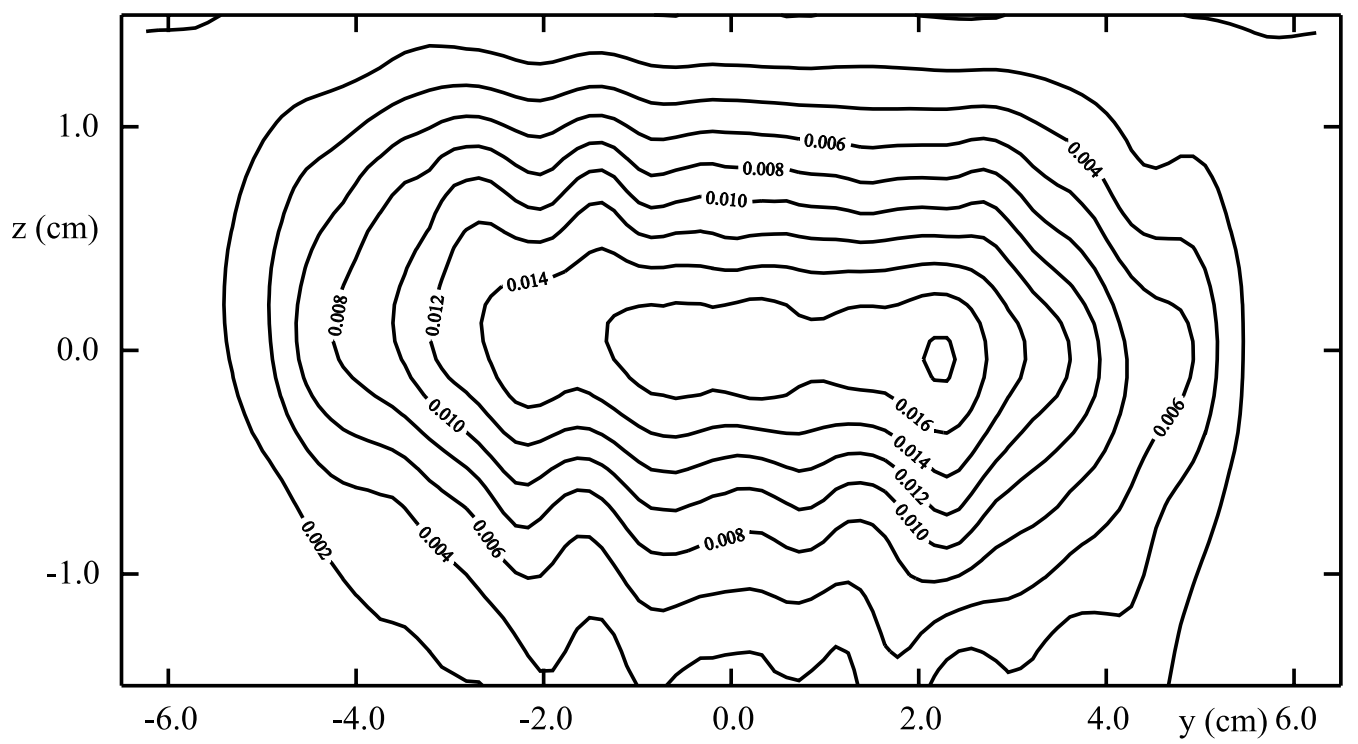

Fig. 17. Mean gas fraction contour lines: $x=420 \mathrm{~mm}$.

Here, no confusion should be made with the co-ordinate system. Since the model is to be applied to one plume - subjected to the action of the other - the origin of the co-ordinate system is the gas source point.

In literature, so many different values have been recommended for $\lambda$ that the only sure conclusion seems to be that $\lambda$ lies in the interval $[0,1]$. Ditmars and Cederwall (1974) and Brevik 
(1977) have used $\lambda=0.2$; Fannelop and Sjoen (1980) have used $\lambda=0.65$ whereas Milgram (1983) has used $\lambda=0.8$. Here, we have decided to approach the problem moderately and choose $\lambda=0.6$.

The local mean gas fraction $f(r, x)$ is given by

$$
f(r, x)=\frac{\rho_{\mathrm{a}}-\rho_{\mathrm{m}}(r, x)}{\rho_{\mathrm{a}}-\rho_{\mathrm{g}}(x)},
$$

where $\rho_{\mathrm{g}}(x)$ is the local gas density.

The liquid volume flux and the liquid momentum flux at any elevation are given respectively by

$$
Q=\int_{0}^{\infty} 2 \pi u r \mathrm{~d} r=\pi u_{\mathrm{m}} b^{2}
$$

and

$$
M=\int_{0}^{\infty} 2 \pi \rho_{\mathrm{a}} u^{2} r \mathrm{~d} r=\frac{\pi}{2} \rho_{\mathrm{a}} u_{\mathrm{m}}^{2} b^{2} .
$$

The entrainment assumption is now taken as the explicit starting point of our theory, i.e., it is considered that the inflow velocity at the periphery of the plume is a fraction $\alpha(\theta)$ of the maximum plume centreline velocity according to

$$
\frac{\mathrm{d} Q}{\mathrm{~d} x}=\int_{0}^{2 \pi} \alpha(\theta) u_{\mathrm{m}} b \mathrm{~d} \theta=\alpha_{\text {int }}\left(u_{\mathrm{m}} b\right) .
$$

Please note that in the above equation the entrainment co-efficient, $\alpha$, is allowed to vary along the periphery of the plume with the angle $\theta$ (see Fig. 1 for the geometrical definition of $\theta$ ).

Then, using Boussinesq approximation, and after Ditmars and Cederwall (1974), the equations of conservation of liquid and gas and the equation resulting from equating the buoyancy per unit height to the spatial rate of momentum flux can be written as

$$
\begin{aligned}
& \frac{\mathrm{d}\left(\pi u_{\mathrm{m}} b^{2}\right)}{\mathrm{d} x}=\alpha_{\text {int }}\left(u_{\mathrm{m}} b\right), \\
& \pi \Delta \rho_{\mathrm{m}} \lambda^{2} b^{2}\left(\frac{u_{\mathrm{m}}}{1+\lambda^{2}}+u_{\mathrm{b}}\right)=\frac{\rho_{\mathrm{a}} q_{0} H_{0}}{\left(H_{0}+H-x\right)}, \\
& \frac{\mathrm{d}\left(u_{\mathrm{m}}^{2} b^{2}\right)}{\mathrm{d} x}=2 g \frac{\Delta \rho_{\mathrm{m}}}{\rho_{\mathrm{a}}} \lambda^{2} b^{2},
\end{aligned}
$$

where $q_{0}$ denotes the volume rate of air discharged at atmospheric pressure, $u_{\mathrm{b}}$ the velocity of the air bubbles relative to the gross plume velocity, $H$ the depth above the air source, $H_{0}$ the piezometric head equivalent of atmospheric pressure and $g$ the gravity acceleration.

The results of Barbosa (1997) showed that for the present experimental conditions the diameter of the bubbles ranged from 4 to $19 \mathrm{~mm}$; of these, most bubbles had a diameter between 5 and 11 $\mathrm{mm}$. Barbosa (1997) also showed that most of the bubbles rised with velocities between 0.35 and $1.1 \mathrm{~m} / \mathrm{s}$ with a slip velocity of $0.315 \mathrm{~m} / \mathrm{s}$. When bubbles with a diameter between 1 and $10 \mathrm{~mm}$ rise in still water it is known that the terminal velocity lies between 0.20 and $0.30 \mathrm{~m} / \mathrm{s}$. Thus, because most of the bubbles occurring in the present experiment are large and relatively fast moving, and because most authors have adopted in their work $u_{\mathrm{b}}=0.30 \mathrm{~m} / \mathrm{s}$, we will also adopt this value here. 
Table 2

Plume deflection angle

\begin{tabular}{lllllll}
\hline & Case & & & & & \\
\cline { 2 - 7 } & $\mathrm{i}$ & $\mathrm{j}$ & $\mathrm{k}$ & 1 & $\mathrm{~m}$ & $\mathrm{n}$ \\
\hline$\phi_{\exp }$ & 2.9 & 3.0 & 1.1 & 2.3 & 1.1 & 1.7 \\
$\phi_{\text {theor }}$ & 2.5 & 3.2 & 2.3 & 2.4 & 2.3 & 2.3 \\
\hline
\end{tabular}

Now, we consider that the bending of the plume is primarily due to an inhibition of the entrainment co-efficient in the part of the plume which is nearer to the other. We also consider that all transversal momentum entrained in the plume is retained by its elements as they rise. If $M_{\mathrm{c}}$ denotes the transversal entrained momentum, then

$$
M_{\mathrm{c}}=\int_{0}^{x} \int_{0}^{2 \pi} \rho_{\mathrm{a}}\left(\alpha(\theta) u_{\mathrm{m}} b\right) \alpha(\theta) u_{\mathrm{m}} \cos (\theta) \mathrm{d} \theta \mathrm{d} x
$$

and the plume deflection angle can be approximated by $\tan (\phi)=M_{\mathrm{c}} / M$.

In the present work, $\alpha(\theta)$ has been approximated by

$$
\begin{aligned}
& \alpha(\theta)=\frac{\alpha_{\max }-\alpha_{\min }}{\pi} \theta+\alpha_{\min }, \quad 0<\theta<\pi, \\
& \alpha_{\min }=0.06-0.00115 W_{\mathrm{e}}, \quad \alpha_{\max }=0.065 .
\end{aligned}
$$

We have, thus, proposed a very simple linear correlation for the prediction of the entrainment coefficient behaviour.

The results are shown in Table 2.

It is clear from Table 2 that the results are good provided $W_{\mathrm{e}}$ number is not too small. In fact, cases $k$ and $m$ were the only ones where the model did not show to be appropriate. Under those conditions, $W_{\mathrm{e}}$ is really small and difficult to incorporate into the theory.

\section{Concluding remarks}

The present work has established a firm connection between the bending angle of a bubble plume and the values of $F_{\mathrm{r}}$ and $W_{\mathrm{e}}$ numbers. The work has been important in identifying some relevant parameters to the problem and in working out a strategy to determine values of the entrainment co-efficient in future more sophisticated formulations of the problem. Also, a very simple theory based on integral methods has been advanced which provides good results.

Of course, a limitation of the present theory is its inability no capture any unsteadiness occurring in the flow. As such, the present predictions should be compared with steady data flow. Because the measurements were taken over intervals of 3-4 min, it is generally considered that the theoretical consideration of steady flow is well reproduced by the experimental data. In fact, no significant wandering motion of the plume was observed which makes us presume that all mean data can confidently be used to validate the theory. 


\section{Acknowledgements}

Several people have greatly contributed for a good term of this work. Prof. L.J.S. Bradbury designed the first electronic gadgets that gave origin to the present instrumentation. Dr. J.R. Barbosa Jr. took the first measurements in the experimental rig, having largely improved the hardware and software packages. Dr. P.P.M. Menut helped to initiate the current investigation; he obtained the first data on the double plume geometry. The first author thanks the Brazilian National Research Council (CNPq) for the award of a research fellowships according to Project no. 831024/98-4. The work was financially supported by the CNPq through grant no. 350183/93-7. A short version of this article was presented under the same title at the International Symposium on Multiphase Flow and Transport Phenomena, Antalya, Turkey, September 2000.

\section{References}

Barbosa, J.R.J., 1997. The electro-resistivity method applied to bubble plumes. M.Sc. Thesis, Mech. Eng. Dept., Federal University of Rio de Janeiro.

Barbosa, J.R.J., Bradbury, L.J.S., 1996. Experimental investigations in round bubble plumes. In: Proc. 6th Brazilian National Meeting on Thermal Sciences (ENCIT), Florianopolis, pp. 1073-1078.

Brevik, I., 1977. Two-dimensional air-bubble plume. J. Waterway, Port, Coastal Ocean Div., ASCE 103, $101-115$.

Brevik, I., Killie, R., 1996. Phenomenological description of the axisymmetric air-bubble plume. Int. J. Multiphase Flow 22, 535-549.

Castillejos, A.H., Brimacombe, J.K., 1987. Measurement of physical characteristics of bubbles in gas-liquid plumes. Metall. Trans. B 18B, 649-671.

Chesters, A.K., van Dorrn, M., Goossens, L.H.J., 1980. A general model for unconfined bubble plumes from extended sources. Int. J. Multiphase Flow 6, 499-521.

Ditmars, J.D., Cederwall, K., 1974. Analysis of air-bubble plumes. In: Proc. Coastal Engng. Conf., pp. $2209-2226$ (Chapter 128).

Fannelop, T.K., Sjoen, K., 1980. Hydrodynamics of underwater blowouts. In: Proc. AIAA 18th Aerospace Sci. Meeting, Pasadena, CA.

Herringe, R.A., Davis, M.R., 1974. Detection of instantaneous phase changes in gas-liquid mixtures. J. Phys. E: Sci. Instrum. 7, 807-812.

Joo, S., Guthrie, R.I.L., 1992. Modeling flows and mixing in the steelmaking laddles designed for single- and dual-plug bubbling operations. Metall. Trans. B 23B, 765-778.

Kline, S.J., 1985. The purpose of uncertainty analysis. J. Fluid Engng. 107, 153-160.

Kobus, H.E., 1968. Analysis of the flow induced by air-bubble systems. In: Proc. 11th Coastal Engng. Conf. London, vol. 2, pp. 1016-1031 (Chapter 65).

Kocamustafaogullari, G., Wang, Z., 1991. An experimental study on local interfacial parameters in horizontal bubbly two-phase bubbly flow. Int. J. Multiphase Flow 17, 553-572.

Leung, W.H., Revankar, S.T., Ishii, Y., Ishii, M., 1992. Axial development of interfacial area and void concentration profiles measured by double-sensor probe method. Int. J. Heat Mass Transfer 38, 445-453.

Liu, T.J., Bankoff, S.G., 1993. Structure of air-water bubbly flow in a vertical pipe-II. Void fraction bubble velocity and bubble size distribution. Int. J. Heat Mass Transfer 36, 1061-1072.

Milgram, J.H., 1983. Mean flow in round bubble plumes. J. Fluid Mech. 133, 345-376.

Nassos, G.P., 1963. Development of an eletrical resistivity probe for void fraction in air-water flow, Technical Report 9, Argonne Report ANL-6738.

Neal, L.G., Bankoff, S.G., 1963. A high resolution resistivity probe for determination of local void properties in gasliquid flow. AlChE J. 9, 49-54.

Pera, L., Gebhart, B., 1975. Laminar plume interactions. J. Fluid Mech., 68. 
Reba, I., 1966. Applications of the Coanda effect. Sci. Am. 214, 84-92.

Tacke, K.H., Schubert, H.G., Weber, D.J., Schwerdtfeger, K., 1985. Characteristics of round vertical gas bubble jets. Metall. Trans. B 16B, 263-275.

Teyssedou, A., Tapucu, A., Lortie, M., 1988. Impedance probe to measure local void fraction profiles. Rev. Sci. Instrum. 59, 631-638. 